\title{
VEGFR-3 ligand-binding and kinase activity are required for lymphangiogenesis but not for angiogenesis
}

\author{
Luqing Zhang ${ }^{1, *}$, Fei Zhou ${ }^{1, *}$, Wencan Han $^{1, *}$, Bin Shen ${ }^{1}$, Jincai Luo ${ }^{2}$, Masabumi Shibuya ${ }^{3}$, Yulong He $^{1}$ \\ ${ }^{I}$ Laboratory of Vascular and Cancer Biology, MOE Key Laboratory for Model Animal and Disease Study, Model Animal Research \\ Institute, Nanjing University, 12 Xue Fu Road, Nanjing 210061, China; ${ }^{2}$ Laboratory of Vascular Biology, Institute of Molecular \\ Medicine, Peking University, Beijing 10087, China; ${ }^{3}$ Department of Molecular Oncology, Tokyo Medical and Dental University, \\ Tokyo 113-8519, Japan
}

Although VEGFR-3 deficiency disrupts blood vascular development during early embryogenesis, the underlying mechanism was not clear. To characterize its function in angiogenesis and lymphangiogenesis, we employed two genetically modified mouse models in this study, targeting the coding region for the ligand-binding domain (Vegfr $3^{\triangle L B D}$ ) or the tyrosine kinase domain with an inactivation point mutation $\left(V e g f r 3^{\text {TKmut }}\right)$. We show that lymphatic growth was disrupted in $\operatorname{Veg} f r 3^{\triangle L B D / \triangle L B D}$ and $V e g f r 3^{T K m u t T K m u t}$ mice, but blood vessels developed normally in both embryo and yolk sac. Interestingly, in $V e g f r 3^{\Delta L B D / \triangle L B D}$ but not $V e g f r 3^{\text {TКни } / T \text { ки }}$ mice, lymph sac was present but there was lack of lymphangiogenic sprouting. We further demonstrate that both the wild-type and mutant forms of VEGFR-3 could form heterodimers with VEGFR-2, and decreased the level of phospho-VEGFR-2 and the downstream phospho-Erk1/2 in endothelial cells when they were treated with VEGF-A. These findings indicate that signaling mediated via VEGFR-3 activation by its cognate ligands (VEGF-C/-D) is not required for angiogenesis, and that VEGFR-3 may play a role in this process by modulating VEGFR-2-mediated signals.

Keywords: VEGFR-3; ligand-binding domain; tyrosine kinase; angiogenesis; lymphangiogenesis Cell Research (2010) 20:1319-1331. doi:10.1038/cr.2010.116; published online 10 August 2010

\section{Introduction}

The formation of blood vascular system during embryonic development is a strictly regulated process, with the first formation of primary vascular plexus from the endothelial precursors present in the blood islands of developing embryos, followed by further remodeling into a mature vascular network with a hierarchy of vessels [1-3]. Lymphatic vessel development occurs later than blood vasculature, and initiates by the process of polarized endothelial cell (EC) budding from the cardinal vein after a subpopulation of blood vascular ECs differentiate into lymphatic ECs (LECs) expressing Sox 18 and Prox1 $[1,4-6]$. Various factors have been shown to play a role in the development of blood vascular and lymphatic

\footnotetext{
*These three authors contributed equally to this work. Correspondence: Yulong $\mathrm{He}$

Tel: +86-25-58641512; Fax: +86-25-58641500

E-mail: yhe20005@yahoo.com

Received 25 June 2010; revised 7 July 2010; accepted 12 July 2010; published online 10 August 2010
}

systems. Among them are vascular endothelial growth factors (VEGFs) including five members identified to date in mammals, VEGF, VEGF-B, VEGF-C, VEGF-D, and placenta growth factor. These factors have distinct roles in vascular development by activating their respective receptors known as VEGF receptor-1, -2 , and -3 as well as co-receptors such as heparan sulfate proteoglycans (HSPGs) and neuropilins [1, 7, 8]. VEGF is a key growth factor for blood vessel formation via VEGFR-2, and VEGF-C/-D activate primarily VEGFR-3. After the proteolytic processing, both VEGF-C/-D have shown increased affinities for VEGFR-3, and the fully processed mature forms also bind to and activate VEGFR-2 [9, 10].

The VEGFR-2-mediated signaling pathway plays essential roles in vascular development, including EC proliferation, migration, survival, and arterial-venous specification by the differential regulation of the downstream target activation [7, 8, 11]. VEGFR-3-mediated signals are required for lymphatic vessel growth in development and tumor [12-14]. During the murine development, VEGFR-3 is initially expressed by all the ECs of primary 
vascular network. While VEGFR-3 is expressed mainly in LECs at later stage of embryogenesis and persists in adult lymphatic vessels [15], it could be induced in blood vessels undergoing active angiogenic sprouting, and in some tumor-associated blood vessels [16-19]. The requirement of VEGFR-3 for blood vascular development was first shown by genetic studies showing that mice null for VEGFR-3 die at midgestation (E10.5) before the emergence of lymphatic vessels, resulting from the failure of the remodeling of primary vascular network into a hierarchy of vessels $[20,21]$. However, deletion of VEGF-C, or both VEGF-C/-D, disrupted only lymphatic growth but did not affect blood vascular development [22]. The underlying mechanism for the distinct phenotypes between the mice deficient for VEGFR-3 or its ligands was not clearly understood.

To investigate the biological function of VEGFR-3 in angiogenesis and lymphangiogenesis, we generated a conditional knockout model targeting its ligand-binding domain (LBD) in this study. Mice homozygous for the LBD deletion (Vegfr $3^{\triangle L B D / \triangle L B D}$ ) showed only disruption of lymphatic growth, but blood vascular development in both embryo and yolk sac was not affected. Consistently, using an independent mutant mouse model in which the kinase activity of VEGFR-3 is ablated by a missense mutation in the tyrosine kinase catalytic domain, we have further validated that signaling mediated via the activation of VEGFR3 kinase is only crucial to lymphangiogenesis, but not required for blood vascular development.

\section{Results}

Generation of mice expressing VEGFR-3 $3^{\triangle L B D}$

In this study, we have generated Vegfr $3^{\text {Flox }}$ mice targeting its LBD, including Ig-like domain 2 and part of Ig-like domain 3 encoded by exon 4-6 of Vegfr3 gene (Supplementary information, Figure S1A). To verify that the conditional allele was functional, Vegfr $3^{\text {Flox }}$ mice were crossed with EIIA-Cre mice [23]. Germ-line deletion of the floxed region of Vegfr 3 gene was confirmed by expression analysis of embryos (E10.5) at the mRNA and protein level (Figure 1A and 1B). As shown in Figure 1A, RT-PCR for VEGFR-3 produced two bands with different sizes in the homozygous Vegfr $3^{\triangle L B D / \triangle L B D}$ mice. The bands were further analyzed by DNA sequencing, and the upper band corresponded to the predicted form of VEGFR-3 mRNA missing exon 4-6 coding sequence, which may encode a truncated protein, if any, by exon 1-3 plus part of exon 7 due to a frame shift to introduce a stop codon. Interestingly, the extra lower band resulted from an alternative splicing of VEGFR-3 pre-mRNA missing exon 4-7 coding sequence, which encoded a novel form of VEGFR-3 lacking only the extracellular Ig-like domains 2 and 3 (aa 134-328) corresponding to the speculated ligand-binding region (named VEGFR$3^{\triangle \mathrm{LBD}}$ ). Expression of VEGFR-3 ${ }^{\triangle \mathrm{LBD}}$ protein was confirmed by western blot analysis (Figure 1B). We further showed that VEGFR-3 ${ }^{\triangle \mathrm{LBD}}$ did not have VEGF-C binding activity by co-expressing VEGF-C and VEGFR-3-Ig ${ }^{\triangle L B D}$ (comprised of the full extracellular domains of VEGFR-3 lacking Ig-like domains 2 and 3, and fused with human IgG Fc) in 293E cells. VEGFR-3-Ig consisting of the full extracellular domains plus IgG1 Fc was used as a control (Figure 1C).

Blood vascular network forms normally in mice expressing VEGFR-3 $3^{\triangle L B D}$

To our surprise, we found that mice homozygous for deletion of the LBD ( Vegfr $\left.3^{\triangle L B D / \triangle L B D}\right)$ developed normally at E10.5 (Figure 1D), which was different from the VEGFR-3-deficient mice displaying embryonic lethality at the same stage [20]. There were no obvious defects with the formation of the blood vascular network in both the embryo (head region, Figure 1D; somite region, Supplementary information, Figure 1B) and yolk sac (Figure 1E). Immunofluorescence staining for PECAM-1 and VEGFR-3 further demonstrated that VEGFR-3 or VEGFR-3 ${ }^{\triangle \mathrm{LBD}}$ was expressed by blood vessels in embryos and yolk sac at E10.5 (Figure 1D and 1E), with high expression in the central veins and blood capillaries but low in the intracranial arteries (Figure 1D, white arrow). The intracranial arteries displayed relatively stronger staining for PECAM-1 (Figure 1D, arrow), but were not stained by EphB4, a vein marker, which mainly stained the central vein region (data not shown). VEGFR-3 expression persisted in the yolk sac blood vessels, but was downregulated in those of embryos with only weak staining in blood capillaries by E15.5 (Supplementary information, Figure S2A and S2B). In addition, we found that LYVE1, originally identified as a lymphatic marker [24], was also highly expressed by blood vessels in yolk sac (Figure 1E and Supplementary information, Figure S2B), consistent with the observation by Gordon et al. [25].

We further examined the vascular development in Vegfr $3^{\triangle L B D / \triangle L B D}$ mice at E15.5. As shown in Figure 2A, Vegfr $3^{\triangle L B D / \triangle L B D}$ mice developed severe edema (arrowhead). Whole-mount immunostaining of the skin showed that there was no lymphatic vessel formation in the mice expressing VEGFR-3 ${ }^{\triangle \mathrm{LBD}}$, but blood vascular network formation was not affected (Figure 2A, arrows).

Lymph sac (LS) forms but lymphangiogenic sprouting is disrupted in Vegfr $3^{\triangle L B D / \triangle L B D}$ mice

In spite of the defective lymphatic vessel growth in 
A

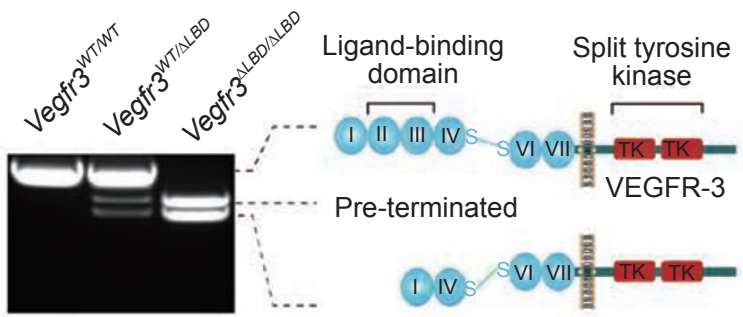

B
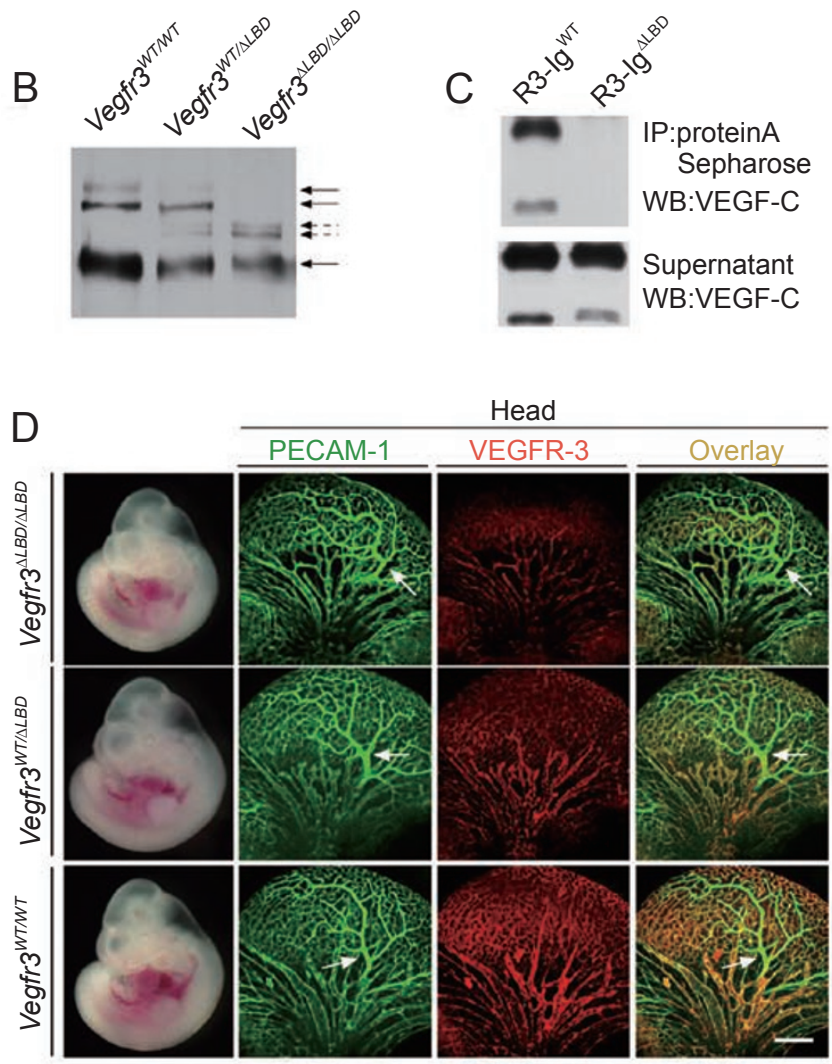

E

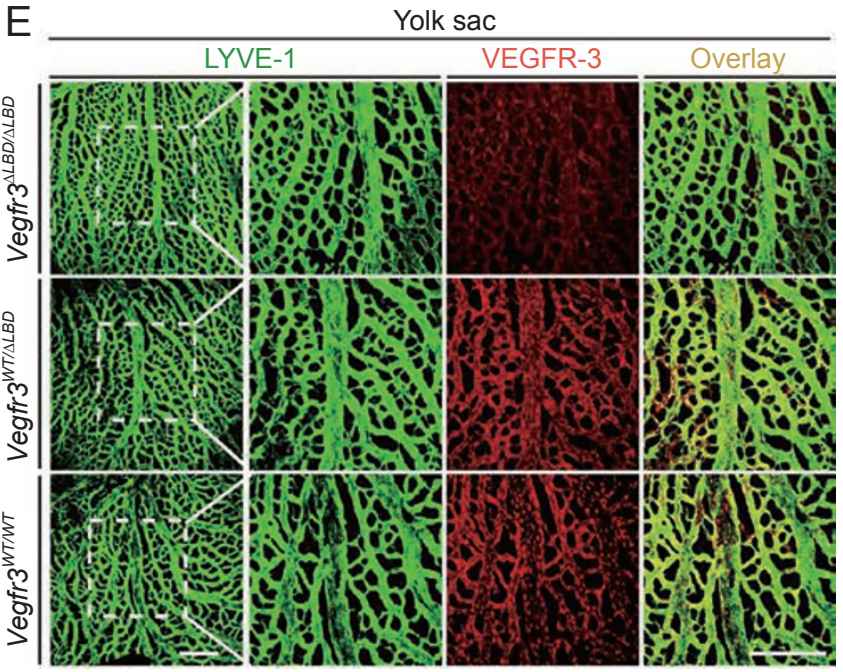

Vegfr $3^{\triangle L B D / \triangle L B D}$ mice, the initial LS formation occurred as examined at E13.5, but displayed an almost round shape (Figure 2B) without lymphatic sprouting in comparison with that in $\operatorname{Veg} f r 3^{W T / \Delta L B D}$ or $\operatorname{Veg} f r 3^{W T / W T}$ mice (Figure 2B, arrows). This may be due to loss of the ability to respond to VEGF-C/-D by LEC expressing VEGFR-3 ${ }^{\triangle \mathrm{LBD}}$. We have further shown that VEGFR-3 $3^{\triangle \mathrm{LBD}}$ has the ability to undergo autophosphorylation in the cultured 293T cells (Supplementary information, Figure S3) and is also weakly detectable in HUVECs (data not shown), while there was no detectable phosphorylation of the kinase inactive form of VEGFR-3 resulting from a point mutation in its kinase region (I1053F, VEGFR-3 ${ }^{\text {TKmut }}$, Supplementary information, Figure S3) [26]. In addition, VEGFR-3 has been shown to interact with other vascular regulators, including integrin $\beta 1$ [27], neuropilin-2 (NRP2, a co-receptor of VEGFR-3) [28], and VEGFR-2 [29]. This suggests that activation of VEGFR-3 kinase can occur in a VEGF-C/-D-independent manner, which may account for the LS formation in $\operatorname{Veg} f r 3^{\triangle L B D / \triangle L B D}$ mice.

Blood vessels develop normally in mice lacking VEGFR-3 kinase activity

To further investigate the biological function of VEGFR-3 in blood vascular development, we used another independent mouse model in which the tyrosine kinase activity was impaired due to the point mutation as described above (VEGFR-3 ${ }^{\text {TKmut }}$ ). The PCR genotyping strategy and the representative result are shown in

Figure 1 Blood vascular network formed normally in embryos expressing the ligand-binding domain (LBD) deleted form of VEGFR-3 (Vegfr $3^{\triangle L B D / \triangle L B D}$ ). (A) Detection of the alternatively spliced variants for VEGFR-3 by RT-PCR. (B) Western blot analysis of VEGFR-3 ${ }^{\text {WT }}$ (WT: wild-type) and VEGFR-3 ${ }^{\triangle L B D}$ protein expression in embryos. Black arrows point to WT VEGFR-3 (two upper bands correspond to the differentially glycosylated full-length VEGFR-3, and the lower band corresponds to the proteolytically processed VEGFR-3 comprised of part of the extracellular domain, transmembrane domain and intracellular kinase domain); dotted black arrows point to the ligandbinding domain-deleted VEGFR-3 (VEGFR-3 ${ }^{\triangle \mathrm{LBD}}$ ). (C) VEGFR$3^{\triangle L B D}$ which lacks only the Ig-like domain 2 and 3 does not bind to its cognate ligand VEGF-C. (D) Analysis of blood vessels in the head region of Vegfr $3^{\triangle L B D / \triangle L B D}$ mice (E10.5-10.75) by wholemount staining for PECAM-1 (green) and VEGFR-3 (red). Blood vascular development in Vegfr $3^{\triangle L B D / \triangle L B D}$ embryos appeared normal compared with the littermates of heterozygous and WT mice. Intracranial arteries were strongly stained by PECAM-1 (white arrows), while VEGFR-3 strongly stained the veins and blood capillaries. Scale bar, $150 \mu \mathrm{m}$. (E) Vegfr $3^{\triangle L B D / \triangle L B D}$ yolk sac had normal blood vasculature in comparison with that of heterozygous and WT mice. Scale bar, $300 \mu \mathrm{m}$. 


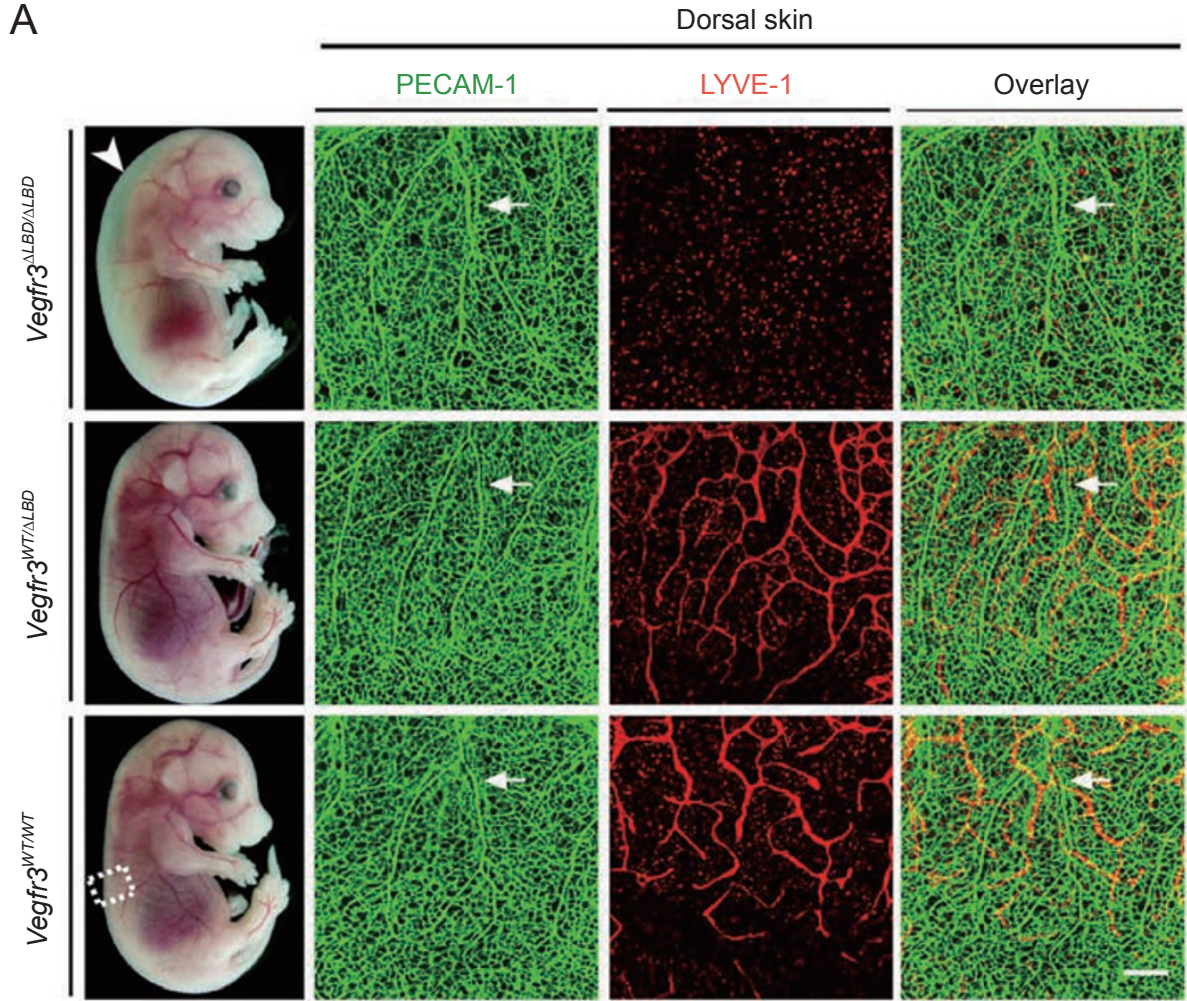

B

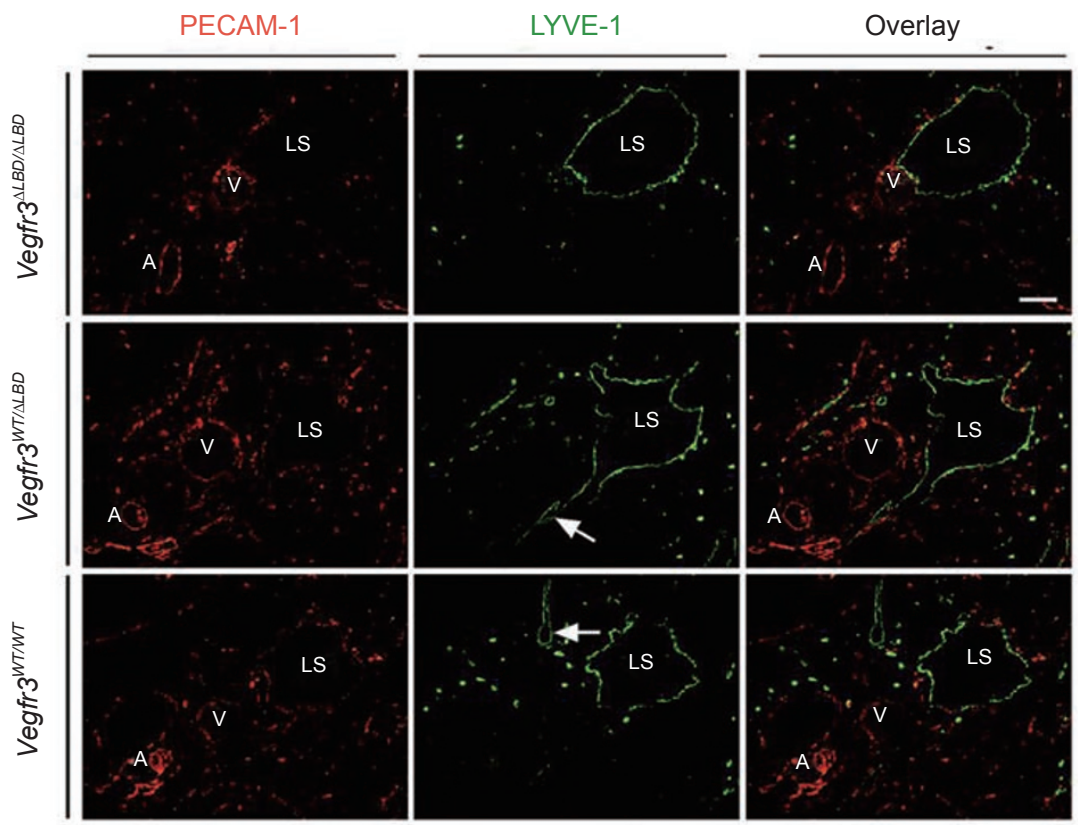

Figure 2 Vegfr $3^{\triangle L B D / \triangle L B D}$ mice showed edema due to lack of lymphatic network formation. (A) Visualization of blood vessels (arrows) and lymphatics in the dorsal skin (dotted white square) by whole-mount immunostaining for PECAM-1 (green) and LYVE-1 (red) in Vegfr3 ${ }^{\triangle L B D / \triangle L B D}$ embryos (E15.5). Note that there is no lymphatic vessel detected in mice homozygous for the LBD deletion and mice show subcutaneous edema (arrowhead). Scale bars, $200 \mu \mathrm{m}$. (B) Sections across E13.5 embryo jugular areas were stained for PECAM-1 (red) and LYVE-1 (green). Jugular lymph sacs (LS) were detected in Vegfr $3^{\triangle L B D / \triangle L B D}$ embryos, but with a round shape, which is different from that of WT and heterozygous mice undergoing active lymphatic sprouting (arrows). A, dorsal aorta; $\mathrm{V}$, jugular vein. Scale bars, $100 \mu \mathrm{m}$. 
Figure $3 \mathrm{~A}$ and $3 \mathrm{~B}$. Western blot analysis revealed that the expression level of VEGFR- $3^{\text {TKmut }}$ is similar to that of WT mice (Figure 3C). Interestingly, mice homozygous for the mutation ( Vegfr $\left.3^{\text {TKmut/TKmut }}\right)$ appeared normal when examined at E10.5 (Figure 3D). Immunohistochemical analysis for PECAM-1 and VEGFR-3 revealed that blood vessels formed in yolk sac, head and somite region in Vegfr $3^{\text {TKmut TKmut }}$ mice, which is comparable to that in WT embryos (Figure 3D and Supplementary information, Figure S4).

We further examined vascular development in Vegfr $3^{\text {TKmut/TKmut }}$ embryos at E15.5. As shown in Figure 4A, severe edema was observed in all the mice homozygous for the tyrosine kinase mutation, and was also seen in approximately half of the heterozygous mice (Vegfr $3^{\text {WT }}$ $\left.{ }_{\text {TKmи }}\right)$. Consistently, the dorsal skin from Vegfr $3^{\text {TKmut/TKmut }}$ mice had the normal hierarchy of blood vasculature as that of WT mice. We also checked the angiogenesis in the retina from Vegfr $3^{\text {WT/WT }}$ and Vegfr $3^{\text {WT/TKmut }}$ mice at postnatal day 5 , and there was no obvious difference in the vessel density and branching points (Supplementary information, Figure S5A-S5C). When syngeneic murine Lewis lung tumor cells were implanted in Vegfr $3^{\text {WT/TKmut }}$ and WT mice, tumor grew at a similar rate and there was no obvious difference in tumor-associated angiogenesis (Supplementary information, Figure S5D). These results are consistent with the vascular phenotype of $\operatorname{Veg} f r 3^{\triangle L B D / \triangle L B D}$ mice, and further validates that signaling mediated via the activation of VEGFR-3 kinase is not required for blood vessel growth.

As expected, there was no lymphatic vessel growth observed in Vegfr $3^{\text {TKmut/TKmut }}$ mice (Figure 4A). In the
A

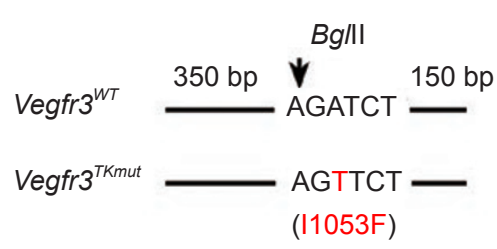

B

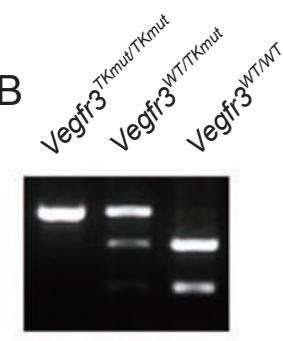

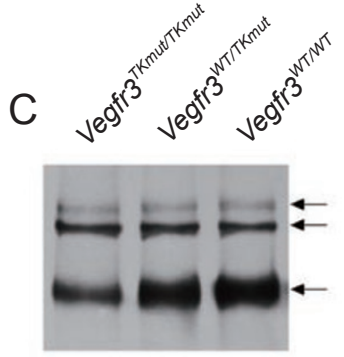

Head

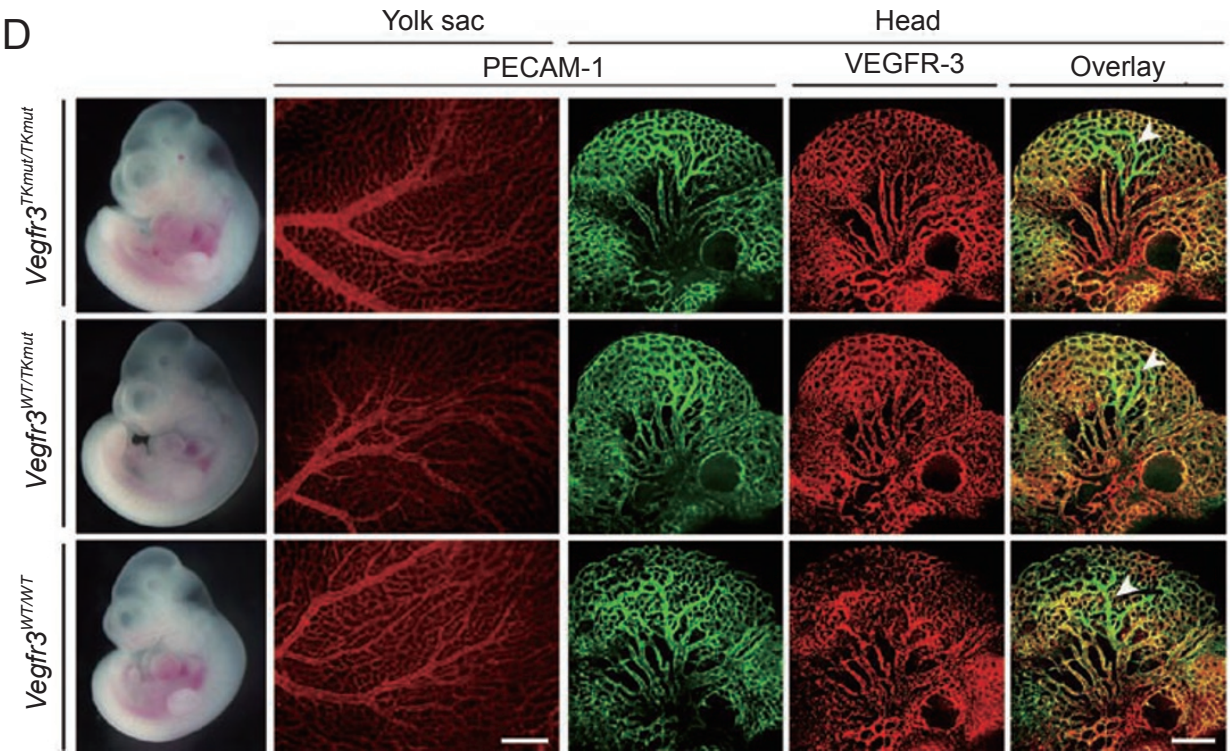

Figure 3 Analysis of blood vascular development in Vegfr3 ${ }^{\text {TKmutTKmut }}$ embryos (E10.5). (A) Genotyping strategy for Vegfr3 ${ }^{\text {TKmut }}$ allele based on the point mutation (I1053F: A to T mutation in exon 23 of VEGFR-3 gene). (B) Representative image of Bg/lldigested PCR products for genotyping of Vegfr3 ${ }^{\text {TKmutTKmut }}$, Vegfr $3^{\text {WTTKmut }}$ and Vegfr ${ }^{\text {WTWT }}$ embryos. (C) Western blot analysis of WT and mutant VEGFR-3 protein level in embryos (E10.5). Arrows point to the VEGFR-3 protein as described. (D) Vegfr3 ${ }^{\text {TKmutTKmut }}$ embryos appeared normal at E10.5. There was no obvious abnormality observed with blood vasculature in yolk sacs stained for PECAM-1 (red). Whole-mount staining with embryos also showed normal vascular development (PECAM-1, green; VEGFR-3, red). Arrowhead indicates intracranial arteries. Scale bars, $300 \mu \mathrm{m}$. 


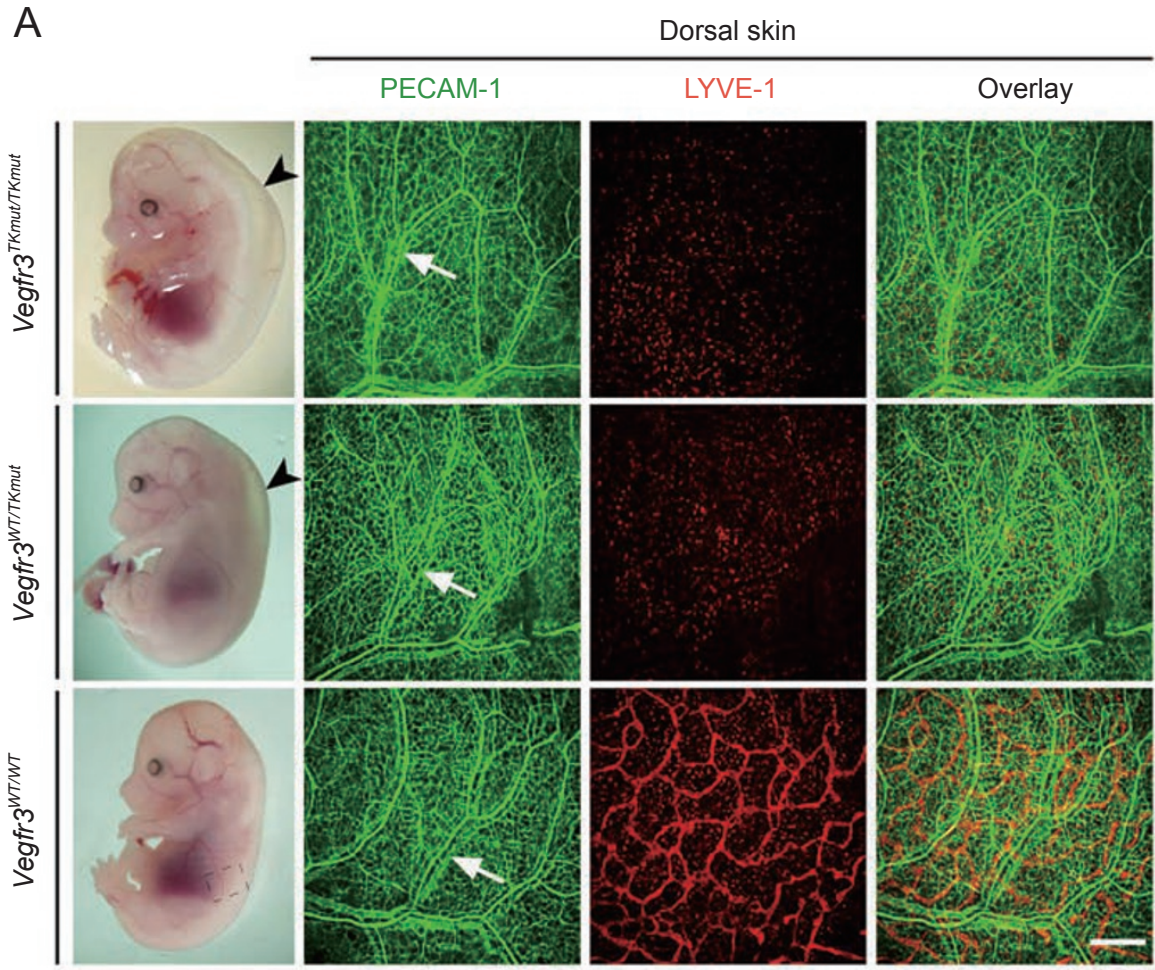

B

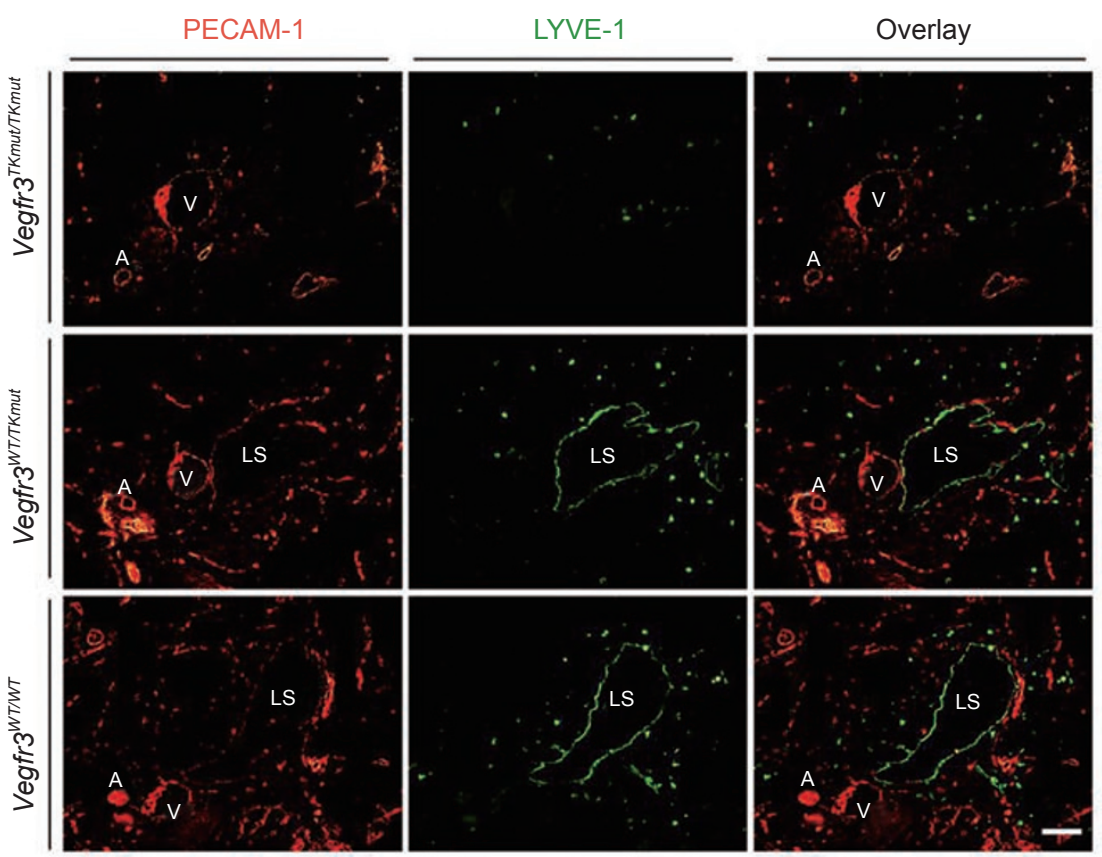

Figure 4 Loss of tyrosine kinase activity of VEGFR-3 disrupted lymph sac formation and lymphatic growth but did not affect angiogenesis (E15.5). (A) All the Vegfr3 $3^{\text {TKmutTKmut }}$ embryos (E15.5) showed severe edema (arrowhead), and about half of the Vegfr $3^{\text {WTTKMmt }}$ embryos also showed similar phenotype (arrowhead). The dorsal skin (dotted square) was stained for PECAM-1 (green) and LYVE-1 (red). Blood vessels (arrows) developed normally while there were no lymphatic vessels detected in the skin of homozygous mutant mice and very few detected in that of heterozygous mice. Scale bars, $300 \mu \mathrm{m}$. (B) E13.5 embryo sections across the jugular region were stained for PECAM-1 (red) and LYVE-1 (green), and jugular lymph sacs (LS) were observed in Vegfr3 ${ }^{\text {WT/TKmut }}$ but not Vegfr3 ${ }^{\text {TKmutTKmut }}$ mice. A, dorsal aorta; V, jugular vein. Scale bars, $100 \mu \mathrm{m}$. 
heterozygous Vegfr $3^{\text {WT/TKmut }}$ mice, there was also no lymphatic vessel growth toward the dorsal central line (Figure 4A), while few enlarged lymphatic vessels around the forelimb skin were occasionally seen (data not shown). There was also no LS formation observed in Vegfr $3^{\text {TKmut }}$ ${ }_{\text {TKmиt }}$ mice at E13.5 (Figure 4D). However, LS formation could be observed in the heterozygous Vegfr $3^{\text {WT/TKmut }}$ mice (Figure 4B), in spite of the decrease in VEGFR-3-mediated signals due to the dominant-negative effect of VEGFR- ${ }^{\text {TKmut }}$. This indicates that the remaining VEGFR-3 signals are sufficient for the primary LS formation, but insufficient for subsequent lymphangiogenesis, leading to the abnormal development of lymphatic system in the heterozygous Vegfr $3^{\text {WT/TKmut }}$ mice (our unpublished data).

\section{VEGFR-3 regulates VEGFR-2-mediated signaling}

To understand the underlying mechanism of the requirement of VEGFR-3 in vascular development, we generated constructs encoding the ligand-binding domain (LBD) deleted (VEGFR-3 ${ }^{\triangle \mathrm{LBD}}$ ), kinase mutated (VEGFR-3 ${ }^{\text {TKmut }}$ ) and WT VEGFR-3, respectively. As shown in Figure 5A, all three forms of VEGFR-3 can interact and be co-precipitated with VEGFR-2 when they were coexpressed in $293 \mathrm{~T}$ cells. This interaction has also been confirmed in HUVECs (data not shown).

To further investigate the consequence of VEGFR-2 and VEGFR-3 interaction, we transduced HUVECs with adenoviral vectors overexpressing each of the three forms of VEGFR-3, and cells were then stimulated with VEGF $_{165}$ recombinant protein for the analysis of the phosphorylated VEGFR-2 level. Shown in Figure 5B is the representative image of five independent experiments. We consistently observed that the phosphorylated VEGFR-2 level was decreased after the overexpression of either the mutant or WT VEGFR-3. This was further confirmed by analysis of the downstream signal mediator, phospho-ERK1/2, which was also decreased as shown in Figure 5C. The findings suggest that VEGFR-3 can modulate EC response to VEGF-A via interaction with VEGFR-2, and that this can be in a ligand-binding or kinase activity-independent way.

The vascular phenotypes of the three genetically engineered mice for VEGFR-3 are summarized in Figure 6A. The potential mechanism of VEGFR-3 function in LS formation and blood vascular growth is proposed in Figure $6 \mathrm{~B}$ and $6 \mathrm{C}$, and a detailed account has been provided in the figure legend.

\section{Discussion}

In this study, we have analyzed the role of VEGFR-3 in angiogenesis and lymphangiogenesis using geneti-

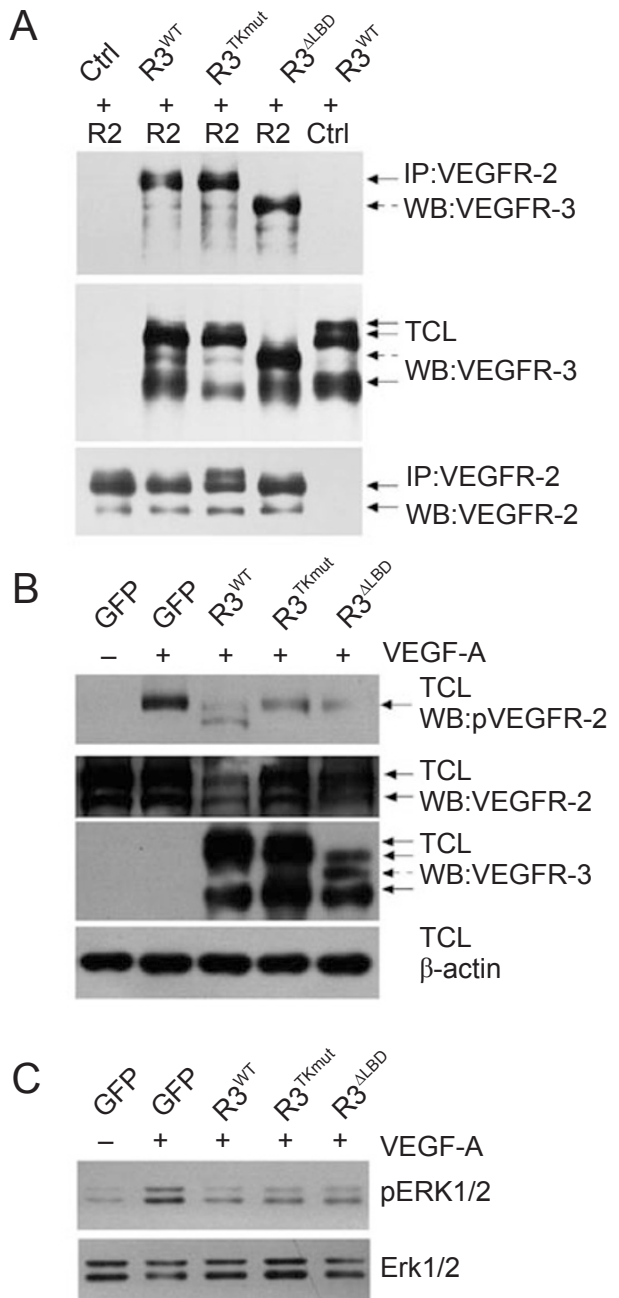

Figure 5 VEGFR-3 heterodimerized with VEGFR-2 to modulate its signaling. (A) All three forms of VEGFR-3 (VEGFR$3^{\mathrm{WT}}$, VEGFR-3 $3^{\triangle \mathrm{LBD}}$ and VEGFR-3 ${ }^{\text {TKmut }}$ ) could interact and be coprecipitated with VEGFR-2. (B) HUVECs were transduced with the recombinant adenoviruses overexpressing the three different forms of VEGFR-3, and then stimulated with VEGF VE5 $_{165}$ as described in Materials and Methods. An equal amount of protein was subjected to western blot analysis for the total and phosphorylated VEGFR-2. (C) The same samples (used in B) were also analyzed for total ERK1/2 and phospho-ERK1/2. IP: immuno-precipitation; WB: western blot; TCL: total cell lysate; Ctrl: control; R2: VEGFR-2; R3: VEGFR-3; arrows point to VEGFR-3 or VEGFR-2 protein; dotted arrows point to the ligand-binding domain-deleted VEGFR-3 (VEGFR-3 ${ }^{\triangle L B D}$ ).

cally modified mouse models targeting its LBD or kinase activity. We show that both mutant forms of VEGFR-3 (VEGFR-3 $3^{\triangle \mathrm{LBD}}$ and VEGFR-3 $3^{\text {TKmut }}$ ) can rescue the blood vascular defects observed in VEGFR-3 null mice [20, 21], but lymphangiogenesis is disrupted in both mutant mice. Interestingly, we have observed that LS formation occurs in mice when the LBD of VEGFR-3 is deleted, 
A

Vascular phenotypes in genetically modified mouse models for VEGFR-3

\begin{tabular}{|c|c|c|c|}
\hline & 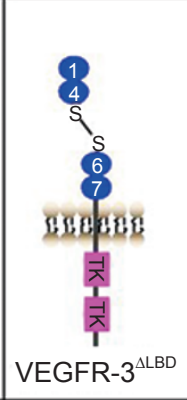 & 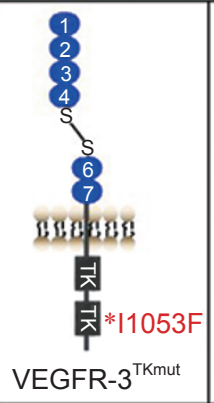 & VEGFR-3 \\
\hline $\begin{array}{l}\text { Angiogenesis } \\
\text { Lymphangiogenesis }\end{array}$ & $\begin{array}{l}\text { Normal } \\
\text { Defective }\end{array}$ & $\begin{array}{l}\text { Normal } \\
\text { Defective }\end{array}$ & $\begin{array}{l}\text { Defective } \\
\text { - }\end{array}$ \\
\hline
\end{tabular}

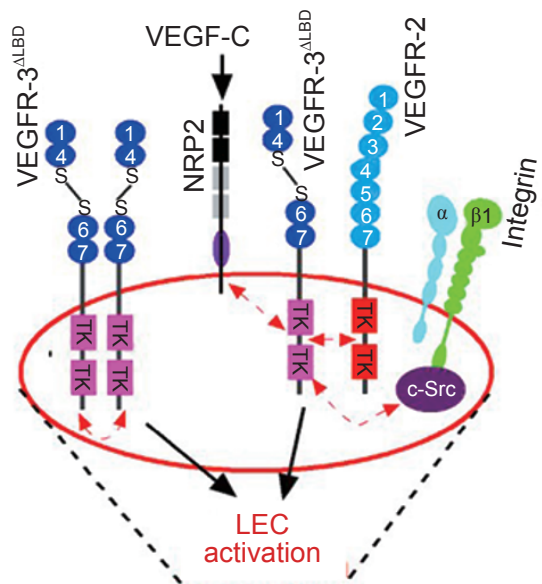

B

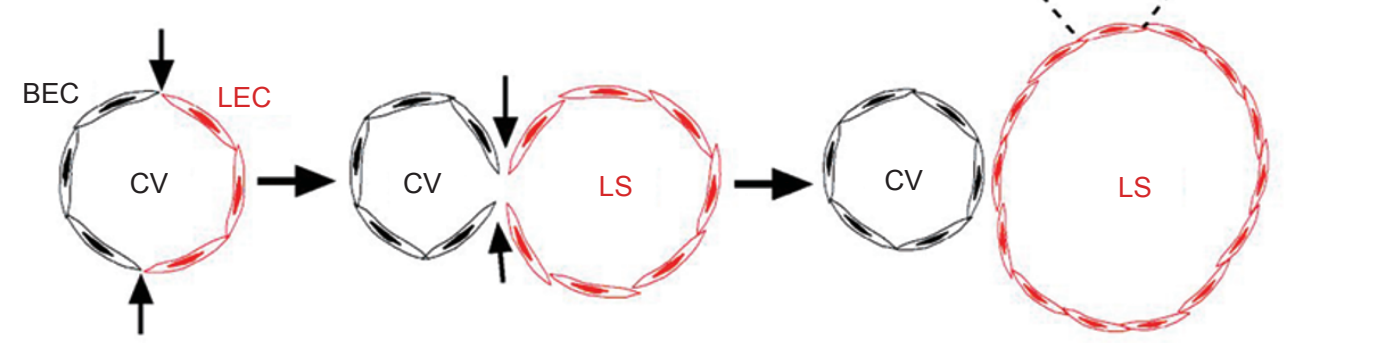

C

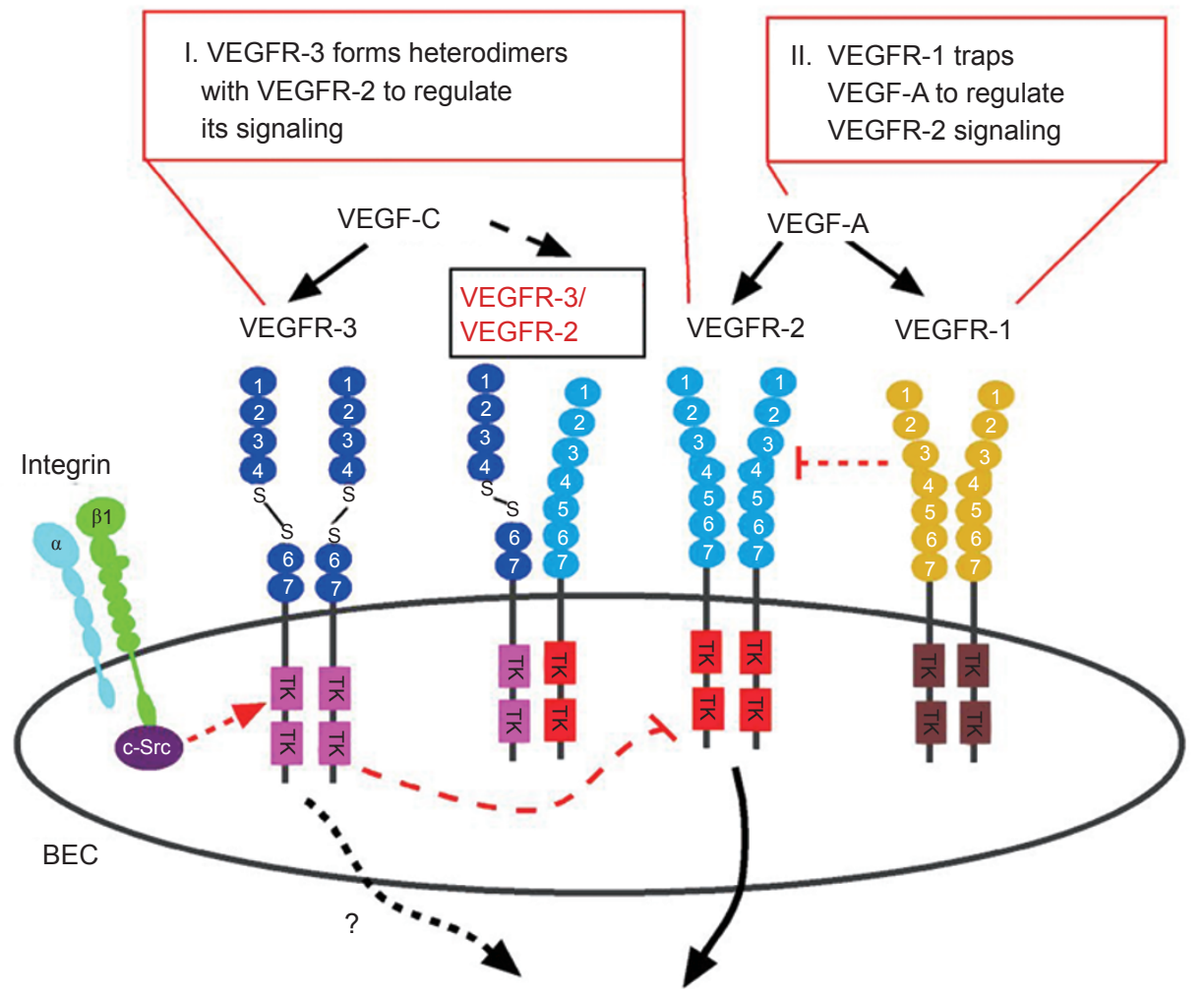

Angiogenesis 
Figure 6 Role and potential mechanism of VEGFR-3 in vascular development. (A) Summary of the vascular phenotypes in the three genetically modified mouse models for VEGFR-3. Loss of the ligand-binding or kinase activity of VEGFR-3 affects only lymphangiogenesis but not angiogenesis. Mice null for VEGFR-3 display abnormal angiogenesis and die before the occurrence of lymphangiogenesis (indicated in the table as -). (B) Lymph sac (LS) formation. LS formation requires VEGFR-3 kinase activity, but is independent of the direct activation of VEGFR-3 by its cognate ligands (VEGF-C/-D). During embryogenesis (around E10 in mouse), a population of blood vascular endothelial cells (BECs) in the cardinal vein (CV) differentiate into lymphatic endothelial cells (LECs). Then LECs dissociate from BECs and form a lymph sac adjacent to the cardinal vein. We have found that VEGFR-3 kinase activity is essential for lymph sac formation as LS does not occur in mice with a kinase-inactive VEGFR-3 (Vegfr3 ${ }^{\text {TKmutTKmut }}$ ). However, lymph sac formation occurs in the absence of ligand-binding activity of VEGFR-3 as shown in Vegfr $3^{\triangle L B D / \triangle L B D}$ mice. It is possible that VEGFR-3 ${ }^{\triangle L B D}$ undergoes the ligand-independent autophosphorylation as shown in Supplementary information, Figure S3. It is also likely that the VEGFR-3 interacting factors, including neuropilin-2 (NRP2, a co-receptor of VEGFR-3), integrin $\beta 1$ or VEGFR-2, could form complexes with VEGFR-3 ${ }^{\triangle L B D}$ and activate its kinase activity for downstream signaling. The exact mechanism of lymph sac formation in Vegfr $3^{\triangle L B D / \triangle L B D}$ mice remains to be investigated. (C) Blood vascular development is independent of VEGFR-3 ligand binding and kinase activity. Shown in this panel is the schematic illustration of the interactions and potential roles of VEGFR-1, VEGFR-2 and VEGFR-3 in the regulation of angiogenesis. VEGFR-1 has been shown to negatively regulate VEGFR-2 signaling by sequestering its ligand VEGF-A. Based on our findings from two genetically modified models, we conclude that VEGFR-3 ligand binding and kinase activity are not required for angiogenesis. At least in cultured endothelial cells (HUVECs), we have found that VEGFR-3 can form heterodimers with VEGFR-2 and regulate its phosphorylation level upon VEGF-A treatment. Therefore, it is possible that VEGFR-3 plays a role in the regulation of blood vessel growth by modulating VEGFR-2-mediated signals. In addition, as VEGFR-3 can interact with other factors as described above, it is also possible that VEGFR-3 could be activated in a way different from that induced by its cognate ligands, and participates in the regulation of blood vascular development. Further studies are required to elucidate the precise mechanism underlying the role of VEGFR-3 in vascular growth.

but this does not happen in mice when the tyrosine kinase activity of VEGFR-3 is ablated. We further demonstrate that the mutant or WT forms of VEGFR-3 can form heterodimers with VEGFR-2 and regulate its phosphorylation level in HUVECs upon VEGF-A treatment. Based on these findings, we conclude that VEGFR-3 activation by its cognate ligands (VEGF-C/-D) is only crucial to lymphangiogenesis but not required for angiogenesis, and propose that VEGFR-3 may play a role in blood vascular development by modulating VEGFR-2mediated signals.

We have found that VEGFR-3 is widely expressed in blood vessels with high expression in blood capillaries and veins but low in arteries during early embryonic development. However, it is not clear whether VEGFR-3 expressed by blood vascular ECs transduces any signals for angiogenesis. Mice deficient of VEGF-C or both VEGF-C/-D appeared normal at E10.5 and did not show any obvious blood vascular defects observed in VEGFR-3 deficient mice [22, 30]. This suggests that the VEGF-C(-D)/VEGFR-3 signaling pathway is not required for blood vascular development. Results obtained from our newly generated genetic model (Vegfr $3^{\triangle L B D / \triangle L B D}$ ) have verified the observation. Our results also exclude the possibility that there might be unidentified cognate ligands binding to/activating the VEGFR-3 pathway in a way similar to that of VEGF-C/-D, which may compensate for the required signals in angiogenic vessel growth. Similar observations have also been made in transgenic mice [12] and in tumor experiments [14, 31], where the soluble VEGFR-3-Ig fusion protein could efficiently block developmental or tumor-associated lymphangiogenesis, but not angiogenesis, by trapping its cognate ligands. Consistently, we have further shown that the blood vascular network also appears normal in mice when the tyrosine kinase activity of VEGFR-3 is ablated. This further validates the above conclusion that signals mediated via VEGFR-3 kinase activation are dispensable for blood vessel growth.

Evidence from extensive studies have shown that the VEGFR-2-mediated signaling pathway plays a crucial role in the regulation of blood vascular network formation, and its activation is strictly regulated at both the ligand and receptor levels [7, 8]. It has been shown that VEGFR-1 negatively regulates VEGFR-2 signaling by sequestering its ligand VEGF [32]. Findings from this study suggest another novel mechanism underlying the regulation of vascular network formation by VEGFR-3, which is independent of its ligand-binding or tyrosine kinase activity when expressed in blood vascular ECs during early embryogenesis. It has been shown that VEGFR-3 can form heterodimers with VEGFR-2 when co-expressed [33], and such VEGFR-2/VEGFR-3 heterodimers could also be detected in vascular ECs in the EB (embryoid body) model by proximity ligation assay [29]. In this study, we have further shown that all the three forms of VEGFR-3 (VEGFR-3 ${ }^{\mathrm{WT}}$, VEGFR$3^{\triangle \mathrm{LBD}}$ and VEGFR-3 ${ }^{\text {TKmut }}$ ) could form heterodimers with 
VEGFR-2 in a ligand-independent manner in cultured cells. Overexpression of either the WT or mutant forms of VEGFR-3 could decrease the level of phosphorylated VEGFR-2 in HUVECs when treated with VEGF-A. This was also validated by the reduced level of its downstream mediator phospho-Erk $1 / 2$ in the same cells. These findings suggest that VEGFR-3 exerts its required function in vascular development indirectly by regulating the level of VEGFR-2 activation, providing an additional layer of control over VEGFR-2-mediated signals. However, it is also possible that VEGFR-3 may function in angiogenesis via interaction with other vascular modulators such as integrin $\beta 1[34,35]$, as proposed in Figure 6. Further in vivo studies are required to verify this. While it has been reported that VEGFR-3 blocking antibodies could suppress angiogenesis in tumor and normal tissues [19], it is likely that the observed angiogenic blocking effects may be due to the interference with VEGFR-2 or other factor-mediated signals in blood vascular ECs.

Finally, interesting observations have also been made regarding VEGFR-3 in lymphatic development. We have confirmed that the extracellular Ig-like domains 2 and 3 of VEGFR-3 are crucial for its ligand-binding and lymphatic development, based on the analysis of Vegfr $3^{\triangle L B D / \triangle L B D}$ mice. Deletion of the LBD of VEGFR-3 disrupted lymphangiogenesis, but LS formation occurred in these mice. There is no LS formation and lymphatic vessel growth observed in Vegfr $3^{\text {TKmut/TKmut }}$ mice. This suggests that the kinase of VEGFR- $3^{\triangle \mathrm{LBD}}$ can still be activated and transduces signals for LEC proliferation and survival after their differentiation from blood vascular ECs, although there is no lymphatic sprouting due to the loss of ligand-sensing ability by LEC expressing VEGFR- $3^{\triangle L B D}$. It is likely that LS formation is independent of the direct activation of VEGFR-3 by its cognate ligands (VEGF-C/-D), and that VEGFR-3 ligands may be required later to provide the spatial cue for lymphatic sprouting from the LS. The exact mechanism of VEGFR$3^{\triangle L B D}$ activation remains to be investigated. It is possible that VEGFR-3 $3^{\triangle L B D}$ undergoes the ligand-independent autophosphorylation as shown in Supplementary information, Figure S3. It is also likely that other VEGFR-3 interacting factors, including NRP2 [28, 36], integrin $\beta 1$ [27], or VEGFR-2 [29], could form complexes with VEGFR-3 ${ }^{\triangle L B D}$ and activate its kinase activity for downstream signaling. However, we have not been able to detect any phospho-VEGFR-3 $3^{\triangle \mathrm{LBD}}$ in embryonic tissues, which may also be due to the fact that the proportion of LECs is small at the stage of LS formation. Therefore, it becomes necessary to isolate the primary LECs expressing VEGFR-3 ${ }^{\triangle \mathrm{LBD}}$ for further analysis.

To summarize, in this study we have successfully generated a mouse model expressing the ligand-binding domain deleted form of VEGFR-3. In combination with another genetic model expressing a kinase inactive form of VEGFR-3, we have shown that the VEGFR-3 ligand binding/kinase activation-mediated signaling pathway is required for lymphangiogenesis but not for angiogenesis. Regulation of vascular development requires a finely coordinated molecular network with dynamic expression/ activation meeting the various cellular needs. In addition to the advanced molecular and cellular tools, more carefully designed genetic models targeting the functional domains or protein interacting sites of vascular regulators are particularly needed to help provide novel insights into the molecular mechanisms underlying blood vascular development.

\section{Materials and Methods}

\section{Generation of Vegfr $3^{\text {Flox }}$ mice and animal work}

All animal experiments were performed in accordance with the institutional guidelines of Model Animal Research Center, Nanjing University. For construction of the Vegfr $3^{\text {Flox }}$ allele, we used the standard BAC retrieval method [37]. Briefly, a 9.6-kb DNA fragment containing the targeting region was retrieved from BAC: BMQ433M21. The first loxP was inserted into intron 3, and the second loxP together with neomycin-resistant gene flanked by FRT sites was inserted into intron 6 . The construct was linearized by NotI and electroporated into R1 embryonic stem cells [38]. Positive ES cell clones were identified by PCR and chimeric mice were generated by microinjection of the ES cells into blastocysts of B6 strain. Genetic transmission was confirmed by backcrossing the chimera with $\mathrm{C} 57 \mathrm{BL} / 6 \mathrm{~J}$ mice and the Neo-cassette was removed using FLPeR mice as previously described [39].

To examine the floxed allele, $\operatorname{Veg} f r 3^{F l o x}$ mice were crossed with EIIA-Cre mice [23] to remove the genomic DNA between the two loxP sites including exon 4-6 of Vegfr 3, which were genotyped by PCR using primers as follows: forward primer (5'-AGA GAC TTC CTG AGC TGT TTC C-3') and reverse primer (5'-AAG CAC AAG TGG TTC TGG GTG C-3') to amplify a 900-bp fragment for the wild-type (WT) allele and a 227-bp fragment for the knockout allele.

Vegfr $3^{\text {WTTKmut }}$ mice were imported from the Medical Research Council Mammalian Genetics Unit Embryo Bank (Harwell, UK), which was generated by ENU (ethylnitrosourea) mutagenesis. Vegfr $3^{\text {WTTKmut }}$ (also named Chy mice) [26] contains a point mutation $(\mathrm{I1053 \textrm {F }})$ in the tyrosine kinase region, which inactivates its kinase activity. Vegfr $3^{\text {WTTKKmut }}$ mice were maintained in $\mathrm{C} 3 \mathrm{H}$ background. As the point mutation ablated a $B g l I I$ restriction site (from AGATCT to AGTTCT), genotyping was performed by amplifying a 495-bp DNA fragment containing the mutation site and then digested by BglII. Primers used are as follows: forward primer: 5'TCC CAT TTT GGA GGA AAC TCA GC-3'; reverse primer: 5'CAA AGG TTC CTG TCA CCT TGA GC-3'. PCR products were digested by $B g l I I$, which produced two bands ( 324 and $171 \mathrm{bp}$ ) with WT mice, while a 495-bp band was obtained from the Vegfr $3^{\text {TKmut } T \text { KKmut }}$ mice. 


\section{Immunostaining}

For whole-mount immunostaining, embryos, yolk sac, embryonic back skin and postnatal retina were harvested, fixed in $4 \%$ paraformaldehyde, blocked with $3 \%(\mathrm{w} / \mathrm{v})$ milk in PBS-TX $(0.3 \%$ Triton X-100), and incubated with primary antibodies overnight at $4{ }^{\circ} \mathrm{C}$. The antibodies used were rat anti-mouse PECAM-1 (BD Pharmingen, 553370) or hamster anti-mouse PECAM-1 monoclonal antibody (Chemicon, MAB1398Z), rabbit anti-mouse LYVE1 (Abcam, ab14917), affinity-purified goat anti-mouse VEGFR-3 (R\&D Systems, AF743), and goat anti-mouse EphB4 (R\&D Systems, AF446). Appropriate Alexa 488, Alexa594 (Invitrogen), Cy3 or Cy5 (Jackson) conjugated secondary antibodies were used for staining. All fluorescently labeled samples were mounted with Vectashield (VectorLabs), and analyzed with a confocal microscope (Leica TCS SP2 or Olympus FluoView 1000), or Leica MZ16F fluorescent dissection microscope.

For section staining, mouse embryos were collected and fixed in $4 \%$ PFA overnight at $4{ }^{\circ} \mathrm{C}$, dehydrated and embedded in paraffin. Consecutive sections $(6 \mu \mathrm{m})$ were incubated with antibodies for PECAM-1 (BD Pharmingen) or LYVE-1 (Abcam), followed by staining with the appropriate fluorochrome-conjugated secondary antibodies and mounted as described above.

\section{Analysis of the alternatively spliced VEGFR-3 forms by RT- PCR}

Embryos (E10.5) were collected and homogenized in Trizol (Invitrogen). RNA extraction and reverse transcription was performed following standard procedures. For PCR amplification of the cDNA fragment coding for the extracellular region of VEGFR-3, the following primers were used: forward primer: $5^{\prime}$-ATC GAA GCT TTC ACT CCC AGC CTA GAG CTG C-3'; reverse primer: 5'-TAC CAG CGC AGA TGT TCG TAC G-3'. PCR products were gel purified, subcloned and followed by DNA sequencing.

\section{DNA constructs}

The full-length VEGFR-3 cDNA was obtained by RT-PCR from the liver tissue of C57BL/6J mice. Briefly two pairs of primers were used for the PCR amplification as follows: For1: 5'-ATC GAA GCT TGA GCT GCG GCC GCG CAG GTC-3'; Rev1: 5'TAC CAG CGC AGA TGT TCG TAC G-3'; and For2: 5'-AAA GTG GGC CAG GAT GAG AGA C-3'; Rev2: 5'-AGC TGA TAT CTC CAC AGA CAA TCA GAT CCA GG-3'. The PCR fragments were subcloned into pcDNA3.1-V5-HisA vector (Invitrogen) to obtain pcDNA3.1-V5-HisA-mVEGFR-3, and the insert was verified by DNA sequencing. To generate the kinase inactive form of VEGFR-3 (VEGFR-3 ${ }^{\text {TKmut }}$ ), we point-mutated the WT VEGFR-3 cDNA following the procedures described in Stratagene's QuikChange ${ }^{\circledR}$ II Site-Directed Mutagenesis method. The primers used were as follows: A3157T-For: 5'-GAA AGT GAC ATA GTG AAG TTC TGC GAC TTT GGC CTC-3' and A3157T-Rev: 5'-GAG GCC AAA GTC GCA GAA CTT CAC TAT GTC ACT TTC-3'. For the generation of LBD-deleted form of VEGFR-3, Vegfr $3^{\triangle L B D / \triangle L B D}$ embryos were collected for RT-PCR amplification of the cDNA fragment encoding the extracellular domain of VEGFR-3 lacking LBD, which was then ligated with the cDNA for the rest part of VEGFR-3 to obtain pcDNA3.1-V5-HisAmVEGFR-3 ${ }^{\triangle \mathrm{LBD}}$. The insert was verified by DNA sequencing. For the production of recombinant adenoviruses, all three forms of
VEGFR-3 (WT, Tkmut and $\triangle \mathrm{LBD}$ ) were subcloned into pShuttleIRES-hrGFP1 (Stratagene).

The full-length VEGFR-2 cDNA (NM 010612.2) was cloned from the mouse tissues as described above using the following primers: mKDR-For1: 5'-GCG GCC GCC ACC ATG GAG AGC AAG GCG CTG CTA GC-3' and mKDR-Rev1: 5'-CTC ATC CAA GGG CAA TTC ATC TG-3' to produce a 5' 2.4-kb fragment, and mKDR-For2: 5'-GGG AAC TGA AGA CAG GCT ACT TGT C-3' and mKDR-Rev2: 5'-CTC GAG AAC AGG AGG TGA GCG CAG TGT GG-3' to produce a 3' $1.6-\mathrm{kb}$ fragment. The two fragments were ligated and subcloned into pcDNA3.1-V5-HisA and pShuttle-IRES-hrGFP1.

To generate soluble forms of VEGFR-3 fused with the human IgG1 Fc domain (VEGFR3-Ig ${ }^{\text {WT }}$ and VEGFR3- Ig $^{\Delta \mathrm{LBD}}$ ), cDNA coding for the full extracellular domain of VEGFR-3 or lacking Ig-like domains 2 and 3 were PCR amplified from the respective constructs as described above. The primers used were as follows: mVEGFR3-For3: 5'-ATC GAA GCT TGA GCT GCG GCC GCG CAG GTC-3' and mVEGFR3-Rev3: 5'-GTC AAG ATC TAC TGC CAC GCT GGC AGA G-3'. PCR products, together with the human IgG1 Fc coding region, were then subcloned into the pcDNA3 expression vector. The full-length human VEGF-C (NM_005429.2) was generated as previously described [40] and subcloned into pShuttle-IRES-hrGFP1 vector.

\section{Cell culture}

HUVECs were isolated from umbilical vein as previously described [41], and cultured in Endothelial Cell Basal Medium plus supplements (Promocell). QBI-293A cells were propagated in DMEM (GIBCO) containing $4.5 \mathrm{~g} / 1$ of glucose, $110 \mathrm{mg} / \mathrm{l}$ of $\mathrm{Na}$ pyruvate, $2 \mathrm{mM}$ glutamine, penicillin $(100 \mathrm{U} / \mathrm{ml})$, streptomycin $(100 \mu \mathrm{g} / \mathrm{ml})$ and $10 \%$ FCS. $293 \mathrm{~T}$ cells were cultured in DMEM with $10 \%$ FCS and $293 \mathrm{E}$ cells in DMEM with $5 \%$ FCS, in addition to the routine supplements. The murine Lewis lung carcinoma (LLC) cell line was obtained from American Type Culture Collection, and maintained in DMEM as specified by the manufacturer.

\section{Adenovirus production}

The recombinant adenoviruses expressing different forms of VEGFR-3 (VEGFR-3 ${ }^{\mathrm{WT}}$, VEGFR-3 ${ }^{\mathrm{TKmut}}$, and VEGFR-3 ${ }^{\mathrm{ALBD}}$ ) and GFP were generated using AdEasy system (Qbiogene, Inc.) following the manufacturer's instruction. Briefly, pShuttle-IREShrGFP1, pShuttle-VEGFR-3 ${ }^{\mathrm{WT}}$-IRES-hrGFP1, pShuttle-VEGFR$3^{\text {TKmut }}$-IRES-hrGFP1, pShuttle-VEGFR-3 ${ }^{\Delta \text { LBD }}$-IRES-hrGFP1, and pShuttle-VEGFR-2-IRES-hrGFP1 were linearized by EcoRI or PmeI, and electroporated into BJ5183 containing pAdEasy1 adenovirus backbone vector. Recombined plasmids were further amplified using DH5 $\alpha$ cells. Purified DNA was first linearized with PacI, and transfected into QBI-293A cells to produce the recombinant adenoviruses.

\section{Transfection, immunoprecipitation, and western blot}

For the study of VEGFR-2 and VEGFR-3 interaction, equal amounts of plasmid DNA were used for transfection of 293T cells using polyethylenimine (PEI, Linear, MW 25000 Polysciences Inc. Cat \#: 23966), and protein analysis was performed within 72 h. Briefly cells were washed with ice-cold PBS and lysed in $1 \mathrm{ml}$ of the lysis buffer $\left(2 \mathrm{mM}\right.$ PMSF, $2 \mathrm{mM} \mathrm{Na}_{3} \mathrm{VO}_{4}, 0.5 \times$ Protease Inhibitor Cocktail Tablets without EDTA from Roche Applied Sci- 
ence, $20 \mathrm{mM}$ Tris- $\mathrm{HCl} \mathrm{pH} 8.0,100 \mathrm{mM} \mathrm{NaCl}, 10 \%$ glycerol, 50 $\mathrm{mM} \mathrm{NaF}, 10 \mathrm{mM} \beta$-glycerolphosphate, $5 \mathrm{mM}$ sodium pyrophosphate, $5 \mathrm{mM}$ EDTA, $0.5 \mathrm{mM}$ EGTA, 1\% NP-40). The cell lysates were incubated at $4{ }^{\circ} \mathrm{C}$ for $0.5 \mathrm{~h}$ with rotation and was clarified by centrifugation. Protein concentration was determined using BCA protein assay kit (PIERCE). Equal amounts of protein were used for analysis and VEGFR-2 was immunoprecipitated using rabbit polyclonal anti-VEGFR-2 antibodies [42] or M2 beads (Sigma A2220) for Flag-tagged VEGFR-2, and western blotted for VEGFR-3 (eBioscience, AFL4).

For VEGF-C and VEGFR-3 binding experiment, 293E cells were transfected with plasmids expressing hVEGF-C plus VEGFR3-Ig ${ }^{\text {WT }}$ or plus VEGFR3-Ig ${ }^{\Delta \text { LBD }}$. Cells were changed to serum free DMEM 1 day after transfection. The supernatant were collected 2 days later, and was analyzed by immunoprecipitation for VEGFR3-Ig using Protein A-Sepharose 4B (ZYMED 10-1141), followed by western blot for VEGF-C [43].

For the analysis of VEGFR-2 phosphorylation, HUVECs were transduced with recombinant adenoviruses expressing the different forms of VEGFR-3 as described above. Cells were cultured for 2 days before starvation for $16 \mathrm{~h}$, followed by stimulation with 50 $\mathrm{ng} / \mathrm{ml}$ recombinant $\mathrm{VEGF}_{165}(\mathrm{R} \& \mathrm{D}, 293-\mathrm{VE})$ for $5 \mathrm{~min}$ at $37{ }^{\circ} \mathrm{C}$. Cells were collected for protein analysis as described above. Antibodies used include mouse monoclonal to VEGFR-2 (A-3, SANTA CRUZ sc-6251); rat monoclonal to mVEGFR-3 (AFL4, eBioscience 14-5998); rabbit monoclonal to phospho-VEGFR-2 Tyr1175 (Cell Signaling Technology \#2478); rabbit monoclonal to Erk1/2 (Cell Signaling Technology \#9102); rabbit monoclonal to phosphoErk1/2 (Cell Signaling Technology \#9101); and mouse monoclonal to $\beta$-actin antibody (C4, SANTA CRUZ sc-47778).

For the analysis of in vivo expression of VEGFR- $3^{\mathrm{WT}}$, VEGFR$3^{\text {TKmut }}$, and VEGFR-3 ${ }^{\triangle \mathrm{LBD}}, \mathrm{E} 10.5$ embryos were collected and homogenized in the lysis buffer as described. Equal amount of protein was used for analysis and VEGFR-3 was immunoprecipitated using goat polyclonal anti-mouse VEGFR-3 antibody (R\&D Systems, AF743). The immunocomplexes were pulled down using Protein G-Sepharose 4B (Zymed, 101242), and analyzed by western blot using the monoclonal rat anti-mouse VEGFR-3 antibody (AFL4, eBioscience).

\section{Xenotransplantation}

Approximately $2.0 \times 10^{6}$ LLC cells in $100 \mu \mathrm{l}$ of PBS were implanted in the s.c. tissue of the back flank region of WT and heterozygous $\operatorname{Vegfr} 3^{\text {WT/TKmut }}$ mice (backcrossed into the C57BL/6JO1aHsd genetic background, 8-10 weeks old). The tumor-bearing mice were sacrificed within 3 weeks, and tissues were collected and processed for histological analysis.

\section{Statistical analysis}

Statistical analysis was performed with the unpaired $t$ test. All statistical tests were two-tailed.

\section{Acknowledgments}

We thank Dr Lena Claesson-Welsh (Uppsala University), and PIs of Model Animal Research Center (MARC, Nanjing University) for the helpful discussion about the work, and Yanlan Cao, Wenting Shi and all the staff in the MARC Animal facility of Nanjing University for excellent technical assistance. This work was financially supported by grants from the National Natural Science Foundation of China (30771069, 30671038, and 30930028), the Ministry of Science and Technology of China (2006CB943500), and the Ministry of Education of China (NCET: Program for New Century Excellent Talents in University).

\section{References}

1 Adams RH, Alitalo K. Molecular regulation of angiogenesis and lymphangiogenesis. Nat Rev Mol Cell Biol 2007; 8:464478.

2 Coultas L, Chawengsaksophak K, Rossant J. Endothelial cells and VEGF in vascular development. Nature 2005; 438:937945.

3 De Val S, Black BL. Transcriptional control of endothelial cell development. Dev Cell 2009; 16:180-195.

4 Wigle JT, Oliver G. Prox1 function is required for the development of the murine lymphatic system. Cell 1999; 98:769778.

5 Oliver G, Detmar M. The rediscovery of the lymphatic system: old and new insights into the development and biological function of the lymphatic vasculature. Genes Dev 2002; 16:773-783.

6 Francois M, Caprini A, Hosking B, et al. Sox18 induces development of the lymphatic vasculature in mice. Nature 2008; 456:643-647.

7 Li X, Claesson-Welsh L, Shibuya M. VEGF receptor signal transduction. Methods Enzymol 2008; 443:261-284.

8 Ferrara N, Gerber HP, LeCouter J. The biology of VEGF and its receptors. Nat Med 2003; 9:669-676.

9 Joukov V, Sorsa T, Kumar V, et al. Proteolytic processing regulates receptor specificity and activity of VEGF-C. EMBO $J$ 1997; 16:3898-3911.

10 Stacker SA, Stenvers K, Caesar C, et al. Biosynthesis of vascular endothelial growth factor-D involves proteolytic processing which generates non-covalent homodimers. $J$ Biol Chem 1999; 274:32127-32136.

11 Lamont RE, Childs S. MAPping out arteries and veins. Sci STKE 2006; 2006:pe39.

12 Makinen T, Jussila L, Veikkola T, et al. Inhibition of lymphangiogenesis with resulting lymphedema in transgenic mice expressing soluble VEGF receptor-3. Nat Med 2001; 7:199205.

13 Karkkainen MJ, Ferrell RE, Lawrence EC, et al. Missense mutations interfere with VEGFR-3 signalling in primary lymphoedema. Nat Genet 2000; 25:153-159.

14 He Y, Kozaki K, Karpanen T, et al. Suppression of tumor lymphangiogenesis and lymph node metastasis by blocking vascular endothelial growth factor receptor 3 signaling. $J$ Natl Cancer Inst 2002; 94:819-825.

15 Kaipainen A, Korhonen J, Mustonen T, et al. Expression of the fms-like tyrosine kinase 4 gene becomes restricted to lymphatic endothelium during development. Proc Natl Acad Sci USA 1995; 92:3566-3570.

16 Valtola R, Salven P, Heikkila P, et al. VEGFR-3 and its ligand VEGF-C are associated with angiogenesis in breast cancer. Am J Pathol 1999; 154:1381-1390.

17 Clarijs R, Schalkwijk L, Hofmann UB, Ruiter DJ, de Waal RM. Induction of vascular endothelial growth factor recep- 
tor-3 expression on tumor microvasculature as a new progression marker in human cutaneous melanoma. Cancer Res 2002; 62:7059-7065.

18 Kubo H, Fujiwara T, Jussila L, et al. Involvement of vascular endothelial growth factor receptor-3 in maintenance of integrity of endothelial cell lining during tumor angiogenesis. Blood 2000; 96:546-553.

19 Tammela T, Zarkada G, Wallgard E, et al. Blocking VEGFR-3 suppresses angiogenic sprouting and vascular network formation. Nature 2008; 454:656-660.

20 Dumont DJ, Jussila L, Taipale J, et al. Cardiovascular failure in mouse embryos deficient in VEGF receptor-3. Science 1998; 282:946-949.

21 Hamada K, Oike Y, Takakura N, et al. VEGF-C signaling pathways through VEGFR-2 and VEGFR-3 in vasculoangiogenesis and hematopoiesis. Blood 2000; 96:3793-3800.

22 Haiko P, Makinen T, Keskitalo S, et al. Deletion of vascular endothelial growth factor (VEGF-C) and VEGF-D is not equivalent to VEGFR-3-null in mouse embryos. Mol Cell Biol 2008; 28:4843-4850.

23 Lakso M, Pichel JG, Gorman JR, et al. Efficient in vivo manipulation of mouse genomic sequences at the zygote stage. Proc Natl Acad Sci USA 1996; 93:5860-5865.

24 Banerji S, Ni J, Wang SX, et al. LYVE-1, a new homologue of the CD44 glycoprotein, is a lymph-specific receptor for hyaluronan. J Cell Biol 1999; 144:789-801.

25 Gordon EJ, Gale NW, Harvey NL. Expression of the hyaluronan receptor LYVE-1 is not restricted to the lymphatic vasculature; LYVE-1 is also expressed on embryonic blood vessels. Dev Dyn 2008; 237:1901-1909.

26 Karkkainen MJ, Saaristo A, Jussila L, et al. A model for gene therapy of human hereditary lymphedema. Proc Natl Acad Sci USA 2001; 98:12677-12682.

27 Zhang X, Groopman JE, Wang JF. Extracellular matrix regulates endothelial functions through interaction of VEGFR-3 and integrin alpha5beta1. J Cell Physiol 2005; 202:205-214.

28 Karpanen T, Heckman CA, Keskitalo S, et al. Functional interaction of VEGF-C and VEGF-D with neuropilin receptors. FASEB J 2006; 20:1462-1472.

29 Nilsson I, Bahram F, Li X, et al. VEGF receptor 2/-3 heterodimers detected in situ by proximity ligation on angiogenic sprouts. EMBO J 2010; 29:1377-1388.

30 Karkkainen MJ, Haiko P, Sainio K, et al. Vascular endothelial growth factor $\mathrm{C}$ is required for sprouting of the first lymphatic vessels from embryonic veins. Nat Immunol 2004; 5:74-80.

31 He Y, Rajantie I, Pajusola K, et al. Vascular endothelial cell growth factor receptor 3-mediated activation of lymphatic endothelium is crucial for tumor cell entry and spread via lymphatic vessels. Cancer Res 2005; 65:4739-4746.

32 Hiratsuka S, Minowa O, Kuno J, Noda T, Shibuya M. Flt-1 lacking the tyrosine kinase domain is sufficient for normal development and angiogenesis in mice. Proc Natl Acad Sci USA 1998; 95:9349-9354.

33 Dixelius J, Makinen T, Wirzenius M, et al. Ligand-induced vascular endothelial growth factor receptor-3 (VEGFR-3) heterodimerization with VEGFR-2 in primary lymphatic endothelial cells regulates tyrosine phosphorylation sites. J Biol Chem 2003; 278:40973-40979.

34 Wang JF, Zhang XF, Groopman JE. Stimulation of beta 1 integrin induces tyrosine phosphorylation of vascular endothelial growth factor receptor-3 and modulates cell migration. $J$ Biol Chem 2001; 276:41950-41957.

35 Galvagni F, Pennacchini S, Salameh A, et al. Endothelial cell adhesion to the extracellular matrix induces c-Src-dependent VEGFR-3 phosphorylation without the activation of the receptor intrinsic kinase activity. Circ Res 2010; 106:18391848.

$36 \mathrm{Xu}$ Y, Yuan L, Mak J, et al. Neuropilin-2 mediates VEGF-Cinduced lymphatic sprouting together with VEGFR3. J Cell Biol 2010; 188:115-130.

37 Liu P, Jenkins NA, Copeland NG. A highly efficient recombineering-based method for generating conditional knockout mutations. Genome Res 2003; 13:476-484.

38 Nagy A, Rossant J, Nagy R, Abramow-Newerly W, Roder JC. Derivation of completely cell culture-derived mice from early-passage embryonic stem cells. Proc Natl Acad Sci USA 1993; 90:8424-8428.

39 Farley FW, Soriano P, Steffen LS, Dymecki SM. Widespread recombinase expression using FLPeR (flipper) mice. Genesis 2000; 28:106-110.

40 Karpanen T, Egeblad M, Karkkainen MJ, et al. Vascular endothelial growth factor $\mathrm{C}$ promotes tumor lymphangiogenesis and intralymphatic tumor growth. Cancer Res 2001; 61:17861790.

41 Baudin B, Bruneel A, Bosselut N, Vaubourdolle M. A protocol for isolation and culture of human umbilical vein endothelial cells. Nat Protocol 2007; 2:481-485.

42 Takahashi T, Yamaguchi S, Chida K, Shibuya M. A single autophosphorylation site on KDR/Flk-1 is essential for VEGFA-dependent activation of PLC-gamma and DNA synthesis in vascular endothelial cells. EMBO J 2001; 20:2768-2778.

43 Tammela T, He Y, Lyytikka J, et al. Distinct architecture of lymphatic vessels induced by chimeric vascular endothelial growth factor-C/vascular endothelial growth factor heparinbinding domain fusion proteins. Circ Res 2007; 100:14681475 .

(Supplementary information is linked to the online version of the paper on Cell Research website.) 